\title{
Термический коэффициент движения резонансного уровня железа в сплавах $\mathrm{Pb}_{1-x-y} \mathrm{Sn}_{x} \mathrm{Fe}_{y} \mathrm{Te}$
}

\author{
(C) Е.П. Скипетров ${ }^{1,2}$, Б.Б. Ковалев ${ }^{1}$, Л.А. Скипетрова ${ }^{1}$, А.В. Кнотько $^{2}$, В.Е. Слынько \\ ${ }^{1}$ Московский государственный университет им. М.В. Ломоносова (физический фракультет), \\ 119991 Москва, Россия \\ ${ }^{2}$ Московский государственный университет им. М.В. Ломоносова (факультет наук о материалах), \\ 119991 Москва, Россия \\ ${ }^{3}$ Институт проблем материаловедения Национальной академии наук Украины, \\ 58001 Черновцы, Украина \\ E-mail: skip@mig.phys.msu.ru
}

Поступила в Редакцию 15 апреля 2019 г.

В окончательной редакции 22 апреля 2019 г.

Принята к публикации 22 апреля 2019 г.

Исследованы фазовый и элементный состав, температурные зависимости удельного сопротивления и коэффициента Холла (диапазон температур $4.2 \leq T \leq 300 \mathrm{~K}$, магнитные поля $B \leq 0.07$ Тл) в сплавах $\mathrm{Pb}_{1-x-y} \mathrm{Sn}_{x} \mathrm{Fe}_{y}$ Те при вариации состава матрицы и концентрации примеси железа вдоль монокристаллических слитков, синтезированных методом Бриджмена-Стокбаргера. Получены распределения олова и железа вдоль слитков, и обнаружены аномальные температурные зависимости коэффициента Холла, связанные с пиннингом уровня Ферми резонансным уровнем железа, расположенным в валентной зоне сплавов. Анализ полученных экспериментальных результатов проведен в рамках модели перестройки электронной структуры, предполагающей движение уровня железа относительно потолка валентной зоны при увеличении концентрации олова и температуры. Определен термический коэффициент движения уровня железа относительно середины запрещенной зоны, и предложены возможные диаграммы перестройки электронной структуры с ростом температуры в сплавах с нормальным спектром $(0.06 \leq x \leq 0.35)$.

Ключевые слова: сплавы $\mathrm{Pb}_{1-x-y} \mathrm{Sn}_{x} \mathrm{Fe}_{y}$ Те, гальваномагнитные эффекты, электронная структура, резонансный уровень железа.

DOI: 10.21883/FTP.2019.11.48440.9141

\section{1. Введение}

В теллуриде свинца глубокие уровни примесей из первой половины ряда переходных $3 d$-металлов ( $\mathrm{Sc}, \mathrm{Ti}$, $\mathrm{V}, \mathrm{Cr}$ ) в основном являются донорными резонансными уровнями [1-7]. В случае примеси скандия резонансный уровень расположен намного выше дна зоны проводимости $E_{c}$ (энергия уровня $E_{\mathrm{Sc}} \approx E_{c}+280$ мэВ при температуре $T=4.2 \mathrm{~K}$ ), а при движении вдоль ряда переходных металлов он постепенно смещается ко дну зоны проводимости. Затем уровень Mn „проваливается“ глубоко в валентную зону и оказывается целиком заполненным электронами, а легирование марганцем приводит к увеличению ширины запрещенной зоны и не влияет на концентрацию свободных носителей заряда $[8,9]$. Наконец, уровни примесей из второй половины ряда $(\mathrm{Fe}, \mathrm{Co}, \mathrm{Ni}, \mathrm{Cu})$, скорее всего, подобно уровням переходных металлов в соединениях $\mathrm{A}^{\mathrm{III}} \mathrm{B}^{\mathrm{V}}$ и $\mathrm{A}^{\mathrm{II}} \mathrm{B}^{\mathrm{VI}}[10]$ возвращаются к краю валентной зоны $E_{v}$ и находятся либо в запрещенной зоне, либо в валентной зоне. В частности, уровень примеси железа оказался первым уровнем, расположенным при $T=4.2 \mathrm{~K}$ в валентной зоне $\mathrm{PbTe,} \mathrm{под} \mathrm{самым} \mathrm{ее} \mathrm{потолком,} \mathrm{энергия} \mathrm{уровня}$ $E_{\mathrm{Fe}}=E_{v}-(16-20)$ мэВ $[11,12]$.

Недавно было показано, что при увеличении концентрации олова в сплавах $\mathrm{Pb}_{1-x-y} \mathrm{Sn}_{x} \mathrm{Fe}_{y} \mathrm{Te}$ резонансный уровень железа по линейному закону движется в глубь „легкой“ валентной зоны $L_{6}^{+}$, постепенно приближаясь к экстремумам „тяжелой“ валентной подзоны в точках $\Sigma$ зоны Бриллюэна [13,14]. При этом предполагалось, что пересечение резонансного уровня железа, стабилизирующего уровень Ферми, с потолком $\Sigma$-зоны так же, как и в случае резонансного уровня индия в $\mathrm{SnTe}[15-$ 17], может привести к значительному увеличению коэффициента Зеебека $S$ и термоэлектрической добротности полупроводника $Z T=\sigma S^{2} T / \kappa(\sigma-$ удельная электропроводность, $\kappa-$ коэффициент теплопроводности) в результате резкого увеличения плотности состояний на уровне Ферми, уширения резонансного уровня и гибридизации примесных и зонных состояний. Однако оценки, основанные на величинах экспериментально определенных композиционных коэффициентов движения уровня железа и потолка $\Sigma$-зоны, показали, что при гелиевых температурах их пересечение должно произойти лишь при концентрациях олова $x=0.6-0.65$. С другой стороны, для термоэлектрических применений полупроводников на основе $\mathrm{PbTe}$ „рабочим“ интервалом температур является диапазон 500-900 K [1820]. Поэтому для определения перспективных с точки зрения термоэлектрической добротности составов сплавов $\mathrm{Pb}_{1-x-y} \mathrm{Sn}_{x} \mathrm{Fe}_{y} \mathrm{Te}$ необходимо иметь информацию не только о композиционных, но и о термических коэффициентах движения уровня железа и $\Sigma$-зоны. 
В настоящей работе исследованы температурные зависимости удельного сопротивления и коэффициента Холла в слабых магнитных полях (диапазон температур $4.2 \leq T \leq 300 \mathrm{~K}$, магнитные поля $B \leq 0.07$ Тл) в образцах из двух монокристаллических слитков $\mathrm{Pb}_{1-x-y} \mathrm{Sn}_{x} \mathrm{Fe}_{y} \mathrm{Te}$ при вариации концентраций олова и железа. Основными целями были определение термического коэффициента движения уровня железа относительно краев энергетических зон, сопоставление полученных результатов с известными литературными данными по движению $\Sigma$-зоны с ростом температуры и построение возможных диаграмм перестройки электронной структуры сплавов при увеличении температуры.

\section{2. Исследованные образцы, методика эксперимента}

Монокристаллические слитки $\mathrm{Pb}_{1-x-y} \mathrm{Sn}_{x} \mathrm{Fe}_{y} \mathrm{Te} 1085$ и 1197 с номинальным содержанием олова и железа $x=0.08, y=0.02$ и $x=0.08, y=0.01$ соответственно были синтезированы вертикальным методом Бриджмена-Стокбаргера из особо чистых компонентов с добавлением карбонильного железа в слиток 1085 и добавлением $\mathrm{FeTe}_{2}$ в слиток 1197. Методика подготовки исходных компонентов и синтеза монокристаллов подробно описана в работе [14]. С помощью струнной резки слитки разрезались перпендикулярно оси роста, совпадающей с точностью до нескольких угловых градусов с кристаллографическим направлением $\langle 111\rangle$, на 24 шайбы толщиной $\sim 1.5$ мм. Номера шайб используются далее как номера исследованных образцов.

Фазовый состав, однородность образцов и распределение олова и железа вдоль слитков исследовались методом рентгенофлюоресцентного микроанализа с использованием сканирующего электронного микроскопа LEO SUPRA 50VP (LEO Carl Zeiss SMT Ltd, Германия), сопряженного с системой микроанализа INCA Energy+ (Oxford Instruments, Англия). Установлено, что основная монокристаллическая фаза, занимающая практически весь объем образцов, является однородной по содержанию олова. При движении от начала к концу слитков (от образца 24 к образцу 2) концентрация олова в слитках 1085 и 1197 монотонно возрастает от $x \approx 0.07$ до $x \approx 0.21$ и от $x \approx 0.06$ до $x \approx 0.15$ соответственно и удовлетворительно описывается следующим выражением [21,22] (рис. 1):

$$
x=x_{0}+A_{1} \exp \left(\frac{L}{t_{1}}\right)+A_{2} \exp \left(\frac{L}{t_{2}}\right),
$$

где $L=h / h_{0}$ - относительная координата шайбы, $h-$ расстояние от начала слитка до центра шайбы, $h_{0}-$ длина слитка, а $x_{0}, A_{1}, A_{2}, t_{1}, t_{2}$ - безразмерные подгоночные параметры.

В отличие от концентрации олова, концентрация примеси железа $y$ довольно медленно увеличивается вдоль слитков, а ее величина, по-видимому, не превышает 0.5 мол\% при ошибке экспериментального опре-

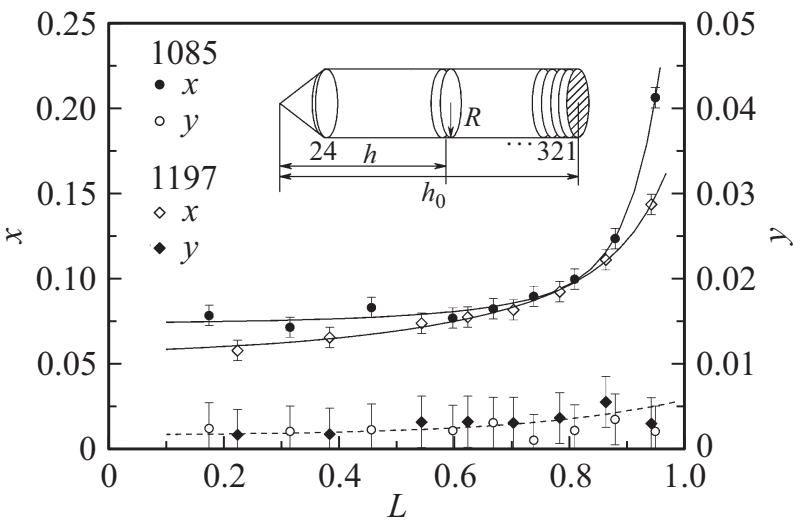

Рис. 1. Модель исследованных монокристаллических слитков и распределения олова и железа по длине слитков $\mathrm{Pb}_{1-x-y} \mathrm{Sn}_{x} \mathrm{Fe}_{y} \mathrm{Te} 1085$ и 1197 (точки - данные рентгенофлюоресцентного микроанализа, сплошные линии - аппроксимация с использованием метода наименьших квадратов формулой (1)).

деления на уровне \pm 0.3 мол\%. Поскольку номинальное содержание железа в ростовой шихте слитков 1085 и 1197 составляло 2 и 1 мол\% и различалось в 2 раза, эти результаты указывают на превышение предела растворимости примеси в обоих слитках. Железо, не растворившееся в основной фазе, входит, по-видимому, в состав микроскопических областей с пониженным содержанием свинца и олова, обогащенных железом, или с химическим составом, близким к соединениям $\mathrm{FeTe}$ или $\mathrm{FeTe}_{2}$, обнаруженным в некоторых образцах из середины и конца слитков [14].

Для исследования температурных зависимостей удельного сопротивления $\rho$ и коэффициента Холла $R_{\mathrm{H}}$ в слабых магнитных полях $(4.2 \leq T \leq 300 \mathrm{~K}, B \leq 0.07$ Тл) четырехзондовым методом на постоянном токе из шайб вырезались образцы в виде прямоугольных параллелепипедов с типичными размерами $4.0 \times 0.7 \times 0.7$ мм. Полученные таким способом образцы травили в растворе брома в бромистоводородной кислоте, затем тщательно промывали этиловым спиртом и дистиллированной водой. Электрические контакты к образцам изготавливали из платиновой или покрытой индием медной проволоки диаметром 0.03 или 0.05 мм соответственно. Токовые контакты припаивали к торцам образцов микропаяльником с использованием припоя In $+4 \% \mathrm{Ag}+1 \% \mathrm{Au}$, а потенциальные контакты приваривались электроискровым методом.

\section{3. Температурные зависимости удельного сопротивления и коэффициента Холла}

Все образцы из слитка 1197 и большая часть образцов из слитка 1085 характеризуются „металлической проводимостью $p$-типа, типичной для нелегированных 
сплавов $\mathrm{Pb}_{1-x} \mathrm{Sn}_{x}$ Те и сплавов с примесями переходных $3 d$-металлов ( $\mathrm{Sc}, \mathrm{Ti}, \mathrm{Cr}, \mathrm{Fe})$, индуцирующими появление резонансных примесных уровней в электронном спектре: при понижении температуры от комнатной до гелиевой удельное сопротивление $\rho$ уменьшается на 1-2 порядка и выходит на насыщение, а коэффициент Холла $R_{\mathrm{H}}$ меняется гораздо слабее и остается положительным во всем исследованном диапазоне температур. При этом холловская подвижность дырок $\mu_{\mathrm{H}}=R_{\mathrm{H}} / \rho$, поведение которой при высоких температурах в основном определяется рассеянием на акустическом деформационном потенциале, увеличивается при понижении температуры не менее чем на порядок, достигая значений на уровне $10^{4} \mathrm{~cm}^{2} /(\mathrm{B} \cdot \mathrm{c})$. Только в нескольких образцах из средней части слитка 1085 на зависимостях $\rho(T)$ появляются дополнительные участки „Полупроводникового“ увеличения сопротивления, связанные, возможно, с резонансным рассеянием дырок и резким уменьшением их подвижности при понижении температуры [14].

Несмотря на это, во всех исследованных образцах температурные зависимости коэффициента Холла имеют один и тот же, аномальный для полупроводников с одним типом носителей заряда, но характерный для сплавов на основе $\mathrm{PbTe}$ с резонансными примесными уровнями, вид (рис. 2). С ростом температуры величина $R_{\mathrm{H}}$ сначала почти не изменяется, а затем постепенно увеличивается в несколько раз. Такое поведение коэффициента Холла уже давно считается прямым доказательством существования резонансного примесного уровня в зоне проводимости или в валентной зоне. Оно объясняется уменьшением концентрации свободных носителей заряда с ростом температуры в результате изменения расстояния между примесным уровнем и краем разрешенной зоны и перераспределения электронов между примесными и зонными состояниями в условиях пиннинга уровня Ферми резонансными уровнями примесей III группы (In, Ga) [23-25], редкоземельных (Yb) [26] и переходных (Cr, Fe) [12,27-30] металлов. Такие аномальные зависимости $R_{\mathrm{H}}(T)$ содержат информацию об изменении положения уровня Ферми, стабилизированного резонансным примесным уровнем, и могут использоваться для определения его основных параметров (энергетического положения при низких температурах, композиционного и термического коэффициентов движения уровня относительно края разрешенной зоны с ростом температуры).

С этой целью мы провели аппроксимацию полученных нами экспериментальных температурных зависимостей коэффициента Холла теоретическими зависимостями, построенными в рамках предложенной ранее модели перестройки электронного спектра сплавов при увеличении содержания олова и температуры $[13,14]$. При этом, во-первых, предполагалось, что во всех образцах уровень Ферми $E_{\mathrm{F}}$ стабилизирован резонансным уровнем железа $E_{\mathrm{Fe}}$, положение которого относительно краев энергетических зон меняется по линейным законам с ростом концентрации олова в сплавах и температуры, и использовалась хорошо известная зависимость ширины запрещенной зоны $E_{g}$ (в мэВ) от состава сплава и температуры [31]:

$$
\begin{gathered}
E_{\mathrm{F}}(x, T)=-\frac{E_{g}(x, T)}{2}-\left[E_{v}-E_{\mathrm{Fe}}(x, T)\right], \\
E_{\mathrm{Fe}}(x, T)=E_{\mathrm{Fe}}(0,0)-\beta x-\alpha T, \\
E_{g}(x, T)=190-543 x+\frac{0.45 T^{2}}{T+50} .
\end{gathered}
$$

Здесь $E_{\mathrm{F}}(x, T)$ - энергия Ферми относительно середины запрещенной зоны $E_{i}, \beta=d\left(E_{i}-E_{\mathrm{Fe}}\right) / d x$ и $\alpha=d\left(E_{i}-E_{\mathrm{Fe}}\right) / d T-$ композиционный и термический коэффициенты движения резонансного уровня железа относительно середины запрещенной зоны.

Во-вторых, считалось, что в широком диапазоне температур (от гелиевой до $\sim 100 \mathrm{~K}$ ) все исследованные образцы являются вырожденными и температурные зависимости коэффициента Холла рассчитывались по зависимостям концентрации дырок от температуры как $R_{\mathrm{H}}(T)=1 / p(T)$, а концентрация дырок $p$ вычислялась в рамках двухзонного закона дисперсии Кейна с параметрами $E_{\perp}=7.1$ эВ, $E_{\|}=0.68$ эВ, приведенными в [31]:

$$
\left(\frac{E_{g}}{2}-E\right)\left(-\frac{E_{g}}{2}-E\right)=E_{\perp} \frac{p_{\perp}^{2}}{2 m_{0}}+E_{\|} \frac{p_{\|}^{2}}{2 m_{0}},
$$

где $E$ - энергия носителей заряда относительно середины запрещенной зоны, $p_{\perp}$ и $p_{\|}-$поперечная и продольная компоненты квазиимпульса, $m_{0}-$ масса свободного электрона.

Учитывалось также, что дырочная поверхность Ферми в сплавах состоит из четырех эллипсоидов вращения с центрами в точках $L$ зоны Бриллюэна, вытянутых вдоль направлений типа $\langle 111\rangle$. Поэтому компоненты квазиимпульса на уровне Ферми $p_{\perp}\left(E_{\mathrm{F}}\right)$ и $p_{\|}\left(E_{\mathrm{F}}\right)$, направленные перпендикулярно и параллельно направлению типа $\langle 111\rangle$, объем одного эллипсоида поверхности Ферми в $p$-пространстве $V_{p}\left(E_{\mathrm{F}}\right)$ и концентрация дырок $p(T)$ вычислялись как

$$
\begin{gathered}
p_{\perp}^{2}\left(E_{\mathrm{F}}\right)=\frac{2 m_{0}}{E_{\perp}}\left(\frac{E_{g}}{2}-E_{\mathrm{F}}\right)\left(-\frac{E_{g}}{2}-E_{\mathrm{F}}\right) \\
p_{\|}^{2}\left(E_{\mathrm{F}}\right)=\frac{2 m_{0}}{E_{\|}}\left(\frac{E_{g}}{2}-E_{\mathrm{F}}\right)\left(-\frac{E_{g}}{2}-E_{\mathrm{F}}\right), \\
V_{p}=\frac{4 \pi}{3} p_{\perp}^{2}\left(E_{\mathrm{F}}\right) p_{\|}\left(E_{\mathrm{F}}\right), \\
p(T)=2 \frac{4 V_{p}}{(2 \pi \hbar)^{3}}=\frac{4\left(2 m_{0}\right)^{3 / 2}}{3 \pi^{2} \hbar^{3} E_{\perp} \sqrt{E_{\|}}}\left(E_{\mathrm{F}}^{2}-\frac{E_{g}^{2}}{4}\right)^{3 / 2} .
\end{gathered}
$$

При аппроксимации экспериментальных температурных зависимостей коэффициента Холла теоретическими кривыми проводилась вариация положения уровня железа $\left(E_{v}-E_{\mathrm{Fe}}\right)$ при низких температурах и величины 

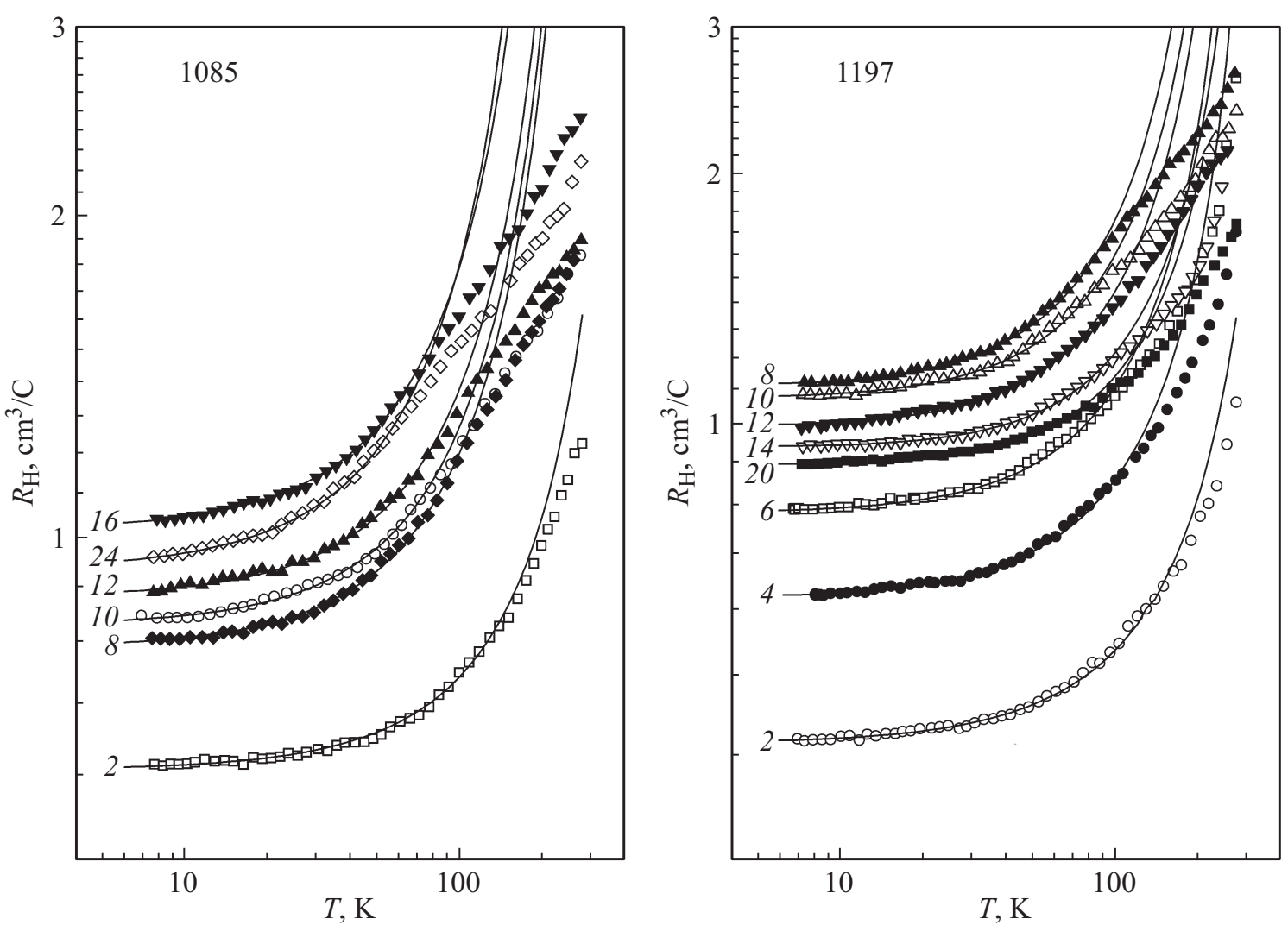

Рис. 2. Температурные зависимости коэффициента Холла в $\mathrm{Pb}_{1-x-y} \mathrm{Sn}_{x} \mathrm{Fe}_{y}$ Те. Номера кривых соответствуют номерам образцов в слитках 1085 и 1197. Точки - экспериментальные данные, линии - аппроксимации экспериментальных зависимостей теоретическими кривыми, рассчитанными в рамках модели перестройки электронной структуры по формулам (2)-(9).

термического коэффициента движения уровня относительно середины запрещенной зоны $\alpha=d\left(E_{i}-E_{\mathrm{Fe}}\right) / d T$ в каждом из исследованных образцов. Полученные таким образом теоретические зависимости $R_{\mathrm{H}}(T)$, хорошо согласующиеся с экспериментальными данными в области низких температур для образцов из обоих исследованных слитков, представлены сплошными линиями на рис. 2. Хорошо видно, что с ростом температуры согласие ухудшается и экспериментальные точки на зависимостях $R_{\mathrm{H}}(T)$ отклоняются вниз от теоретических кривых. Это может быть связано с конечной емкостью примесного уровня железа и нарушением предположений, сделанных нами в рамках описанной выше модели. В частности, при повышении температуры может происходить постепенный переход к невырожденной статистике носителей заряда и в результате собственной генерации и тепловой активации электронов из „тяжелой“ валентной подзоны в „легкую“ (по-другому, активации дырок из ,легкой“ валентной подзоны в „тяжелую“) будет нарушаться предположение об одном типе носителей заряда в исследованных образцах.

На рис. 3 приведены оптимальные для области низких температур значения основных параметров уровня железа для всех исследованных сплавов. Зависимости положения уровня железа относительно потолка валент- ной зоны от состава сплавов практически совпадают с аналогичными зависимостями, полученными ранее по значениям концентраций дырок при $T=4.2 \mathrm{~K}$ в образцах из двух исследованных нами слитков [14]. В обоих слитках точки для образцов со стабилизированным уровнем Ферми хорошо ложатся на общую для обоих слитков прямую, которая при $x=0$ экстраполируется к значению, соответствующему положению резонансного уровня железа в $\mathrm{PbTe}$ (наклонные линии на рис. 3): $E_{v}-E_{\mathrm{Fe}}=16.4+728 x$ мэВ.

Термический коэффициент движения резонансного уровня железа относительно середины запрещенной зоны $(\alpha)$ имеет отрицательный знак во всех образцах, но его абсолютная величина уменьшается с ростом концентрации олова в слитке 1085 и увеличивается в слитке 1197. Однако, если в обоих случаях ограничиться только наиболее надежными точками, соответствующими составам сплавов, при которых наблюдается пиннинг уровня Ферми $(x=0.080-0.100$ в слитке 1085 и $x=0.085-0.145$ в слитке 1197) [14], эта величина почти не меняется при изменении состава сплава и ее среднее значение составляет $\alpha=-(0.07 \pm 0.015)$ мэВ $/ \mathrm{K}$ в интервале концентраций олова $x=0.08-0.145$ (горизонтальные линии на рис. 3). Эта величина в $\sim 3$ раза меньше термического коэффициента движения уровня железа в $\mathrm{PbTe}$, определенного ранее [30], что позволяет 

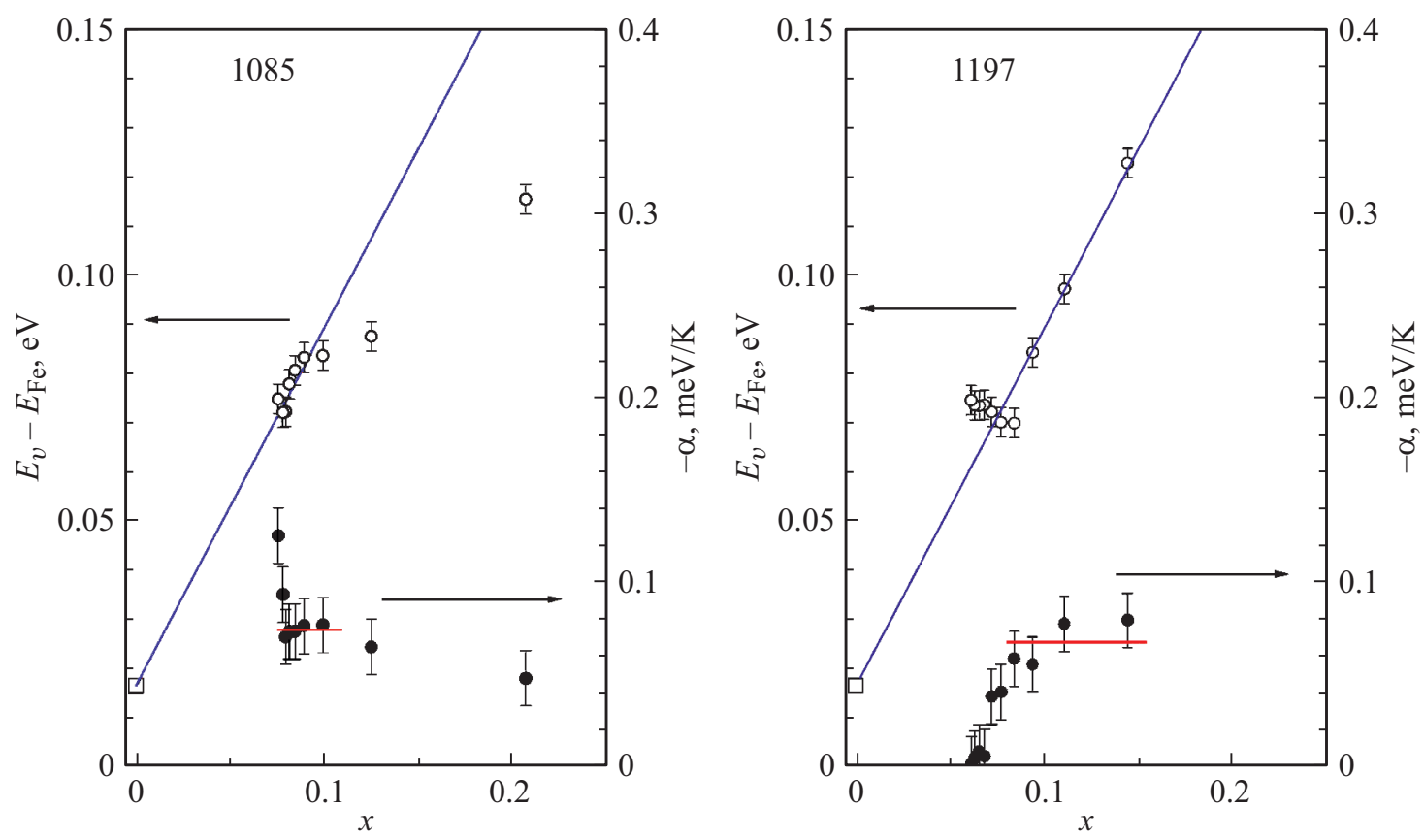

Рис. 3. Зависимости положения уровня Ферми относительно потолка валентной зоны при $T=4.2 \mathrm{~K}$ и термического коэффициента движения уровня железа относительно середины запрещенной зоны от концентрации олова в сплавах $\mathrm{Pb}_{1-x-y} \mathrm{Sn}_{x} \mathrm{Fe}_{y} \mathrm{Te}_{\mathrm{f}}$, рассчитанные в рамках модели перестройки электронной структуры по формулам (2)-(9).

предполагать все-таки уменьшение абсолютной величины $\alpha$ с ростом содержания олова в сплавах.

\section{4. Модели перестройки электронной структуры сплавов при увеличении температуры}

Полученные результаты позволяют нам прогнозировать движение резонансного уровня железа относительно краев энергетических зон и строить возможные модели перестройки электронной структуры сплавов $\mathrm{Pb}_{1-x-y} \mathrm{Sn}_{x} \mathrm{Fe}_{y}$ Te c ростом температуры при вариации концентрации олова в сплавах. На рис. 4 представлены четыре энергетические диаграммы движения уровня железа $E_{\mathrm{Fe}}$ и краев энергетических зон в точках $L$ и $\Sigma$ зоны Бриллюэна при увеличении температуры в сплавах разного состава. Первые две диаграммы относятся к диапазону составов, исследованному в настоящей работе, а последующие две - к большим концентрациям олова в сплавах вплоть до точки инверсии зон в $L$.

При построении этих диаграмм использовалась описанная выше модель перестройки электронного спектра сплавов (см. (2)-(5)) с параметрами $\beta=d\left(E_{i}-E_{\mathrm{Fe}}\right) / d x$ $\approx 4.6$ мэВ/мол\% и $\alpha=d\left(E_{i}-E_{\mathrm{Fe}}\right) / d T \approx-0.07 \mathrm{мэB} / \mathrm{K}$, определенными в работах $[13,14]$ и в настоящей работе, а также данные по движению потолка $\Sigma$-зоны при увеличении содержания олова в сплавах [31] и температуры [31-35]. Необходимо отметить, что если характер движения $\Sigma$-зоны с ростом концентрации олова в сплавах хорошо известен $(\Sigma$-зона движется относительно середины запрещенной зоны со скоростью $d\left(E_{i}-E_{\Sigma}\right) / d x \approx 2.15$ мэВ/мол\% [31]), то с ее поведением при увеличении температуры ситуация более сложная. Долгое время считалось надежно установленным, что при увеличении температуры экстремум „тяжелой“ валентной зоны в РbТе движется практически параллельно краю зоны проводимости $L_{6}^{-}$(прямые 1 на рис. 4), быстро сближается с экстремумом ,легкой“ валентной зоны $L_{6}^{+}$, пересекает его при $T \approx 450 \mathrm{~K}$ и становится основным экстремумом валентной зоны [31,32]. Однако в последнее время эта казавшаяся давно устоявшейся модель подвергается серьезным сомнениям [33-35]. Анализ давно известных экспериментальных данных и результатов современных гальваномагнитных и термоэлектрических исследований сильно легированного $p$-РbТе позволил предположить, что „тяжелая“ валентная подзона сближается с ,легкой“ подзоной примерно вдвое медленнее, двигаясь с ростом температуры почти параллельно середине запрещенной зоны (прямые 2 на pис. 4). В этом случае РbТе должен оставаться прямозонным полупроводником практически во всем „рабочем“ с точки зрения термоэлектрических применений температурном диапазоне, а пересечение валентных подзон в $\mathrm{PbTe}$ должно произойти лишь при 850 вместо $450 \mathrm{~K}$.

На диаграммах хорошо видно, что во втором варианте движения $\Sigma$-зоны при увеличении температуры уровень железа удаляется от потолка „тяжелой“ валентной подзоны и его пересечение с $\Sigma$-зоной в сплавах с нормальным спектром невозможно. По нашим оценкам, уровень железа может оказаться в ее пределах только в сплавах с $x>(0.60-0.65)$. 

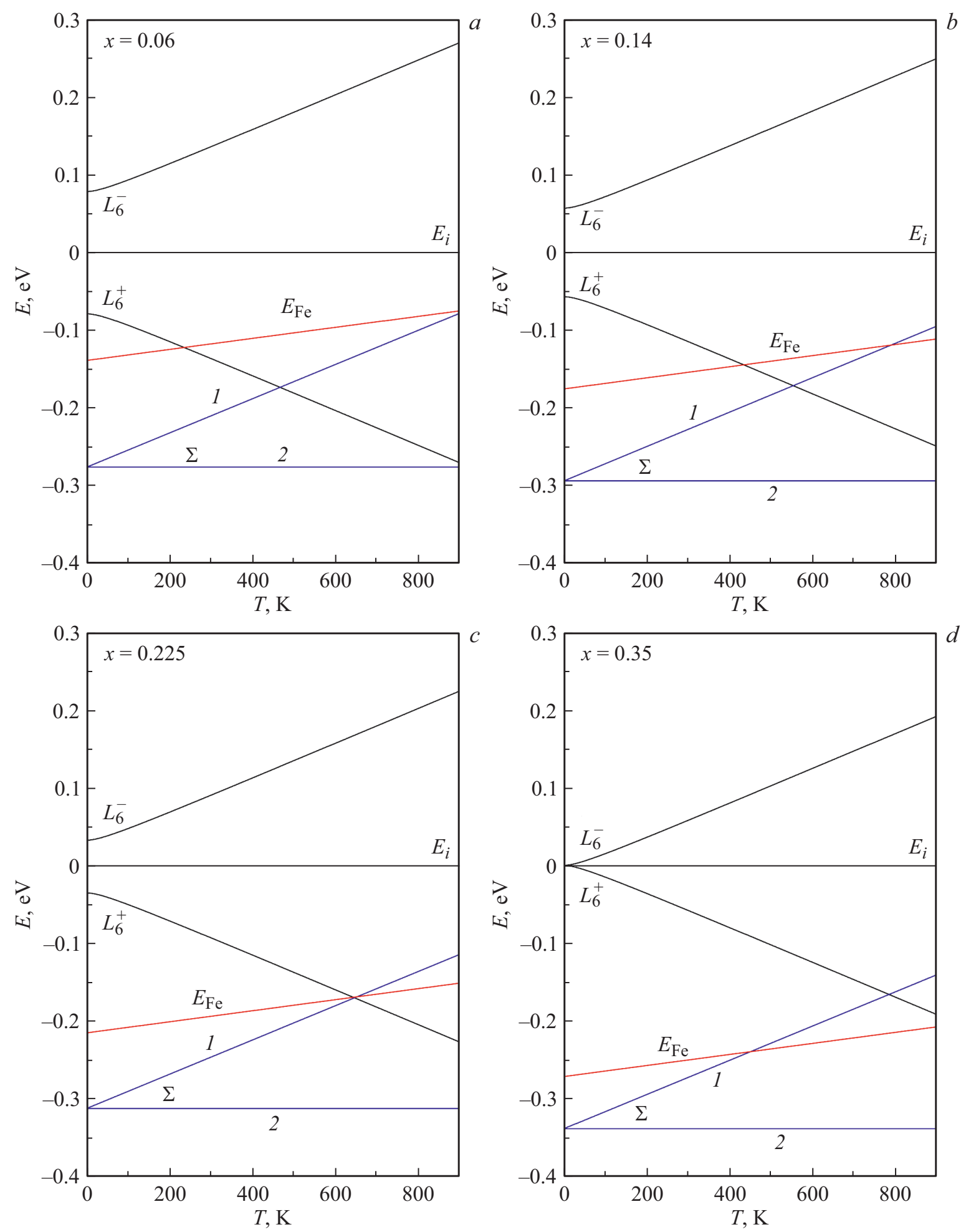

Рис. 4. Возможные диаграммы перестройки электронной структуры сплавов $\mathrm{Pb}_{1-x-y} \mathrm{Sn}_{x} \mathrm{Fe}_{y} \mathrm{Te}$ при увеличении температуры, полученные в рамках модели (2)-(5) с параметрами резонансного уровня железа, определенными в $[13,14]$ и в настоящей работе.

Более благоприятная ситуация реализуется в первом варианте движения $\Sigma$-зоны с ростом температуры (см. рис. 4). В сплавах с содержанием олова от $x=0.06$ до 0.35 точка пересечения уровня железа с ее потолком смещается от 900 к $450 \mathrm{~K}$, т.е. примерно от правой гра- ницы к левой границе „рабочего“ для термоэлектрических приложений температурного интервала. При этом в средней части этого интервала возможно одновременное пересечение уровня железа с краями валентных подзон в точках $L$ и $\Sigma$ зоны Бриллюэна (см. рис. $4, c$ ). Кроме того, 
поскольку на первых трех диаграммах с ростом температуры уровень железа входит в пределы $\Sigma$-зоны из запрещенной зоны, то в случае пиннинга уровня Ферми уровнем железа только при этой критической температуре должен начинаться существенный рост концентрации „тяжелых“ дырок. Поэтому перспективными выглядят также сплавы с содержанием олова вблизи точки инверсии зон в $L(x=0.30-0.35)$, в которых уровень железа пересекает потолок $\Sigma$-зоны при $450-500 \mathrm{~K}$. Затем с ростом температуры должно произойти пересечение краев валентных подзон, а концентрации „тяжелых“ и „легких“ дырок должны увеличиваться и уменьшаться, соответственно вплоть до $900 \mathrm{~K}$ (см. рис. 4,d).

Наконец, отметим, что если, как мы предполагаем, абсолютная величина термического коэффициента движения уровня железа относительно середины запрещенной зоны действительно уменьшается с ростом содержания олова в сплавах, критическая точка пересечения уровня железа с потолком $\Sigma$-зоны на двух последних диаграммах может существенно сдвинуться в сторону низких температур, а диапазон оптимальных составов сплавов может сместиться в более удобную для экспериментальных исследований и вполне достижимую область нормального спектра $(x<0.30)$ (см. рис. 4$)$.

Таким образом, в первом варианте движения $\Sigma$-зоны с ростом температуры, подбирая оптимальный состав матрицы и температурный интервал, в котором предполагается пересечение резонансного уровня железа с потолком „тяжелой“ валентной зоны, можно надеяться на достижение максимального синергетического эффекта повышения термоэлектрической эффективности легированных железом сплавов в результате действия сразу двух основных факторов: присутствия резонансного уровня железа вблизи уровня Ферми и пересечения экстремумов ,легкой“ и ,тяжелой“ валентных подзон в электронном спектре.

\section{5. Заключение}

В образцах из монокристаллических слитков $\mathrm{Pb}_{1-x-y} \mathrm{Sn}_{x} \mathrm{Fe}_{y} \mathrm{Te} \quad(x=0.06-0.21), \quad$ синтезированных методом Бриджмена-Стокбаргера, обнаружены аномальные температурные зависимости коэффициента Холла, указывающие на перераспределение электронов между резонансным уровнем железа и валентной зоной при увеличении температуры в условиях пиннинга уровня Ферми уровнем железа, расположенным в валентной зоне сплавов. В рамках двухзонного закона дисперсии Кейна и модели перестройки электронной структуры сплавов, учитывающей движение уровня железа относительно потолка валентной зоны при увеличении содержания олова и температуры, рассчитаны теоретические температурные зависимости коэффициента Холла, хорошо согласующиеся с экспериментальными при низких температурах. Показано, что абсолютная величина термического коэффициента движения уровня железа относительно середины запрещенной зоны в сплавах с концентрациями олова $x=0.08-0.145$ примерно в 3 раза меньше, чем в $\mathrm{Pb}_{1-y} \mathrm{Fe}_{y} \mathrm{Te}: d\left(E_{i}-E_{\mathrm{Fe}}\right) / d T=$ $=-(0.07 \pm 0.015) \mathrm{мэ} \mathrm{B} / \mathrm{K}$.

Полученные результаты использованы для построения возможных диаграмм перестройки электронной структуры сплавов $\mathrm{Pb}_{1-x-y} \mathrm{Sn}_{x} \mathrm{Fe}_{y}$ Te с ростом температуры в сплавах с нормальным спектром $(0.06 \leq x \leq 0.35)$ и анализа возможности пересечения резонансного уровня железа с потолком „тяжелой“ валентной $\Sigma$-зоны в диапазоне температур 500-900 K.

\section{Финансирование работы}

Работа выполнена при финансовой поддержке Российского фонда фундаментальных исследований (проект № 19-02-00774).

\section{Конфликт интересов}

Авторы заявляют об отсутствии конфликта интересов.

\section{Список литературы}

[1] E.P. Skipetrov, L.A. Skipetrova, A.V. Knotko, E.I. Slynko, V.E. Slynko. J. Appl. Phys., 115, 133702 (2014).

[2] M.N. Vinogradova, E.A. Gurieva, V.I. Zharskii, S.V. Zarubo, L.V. Prokofeva, T.T. Dedegkaev, I.I. Kryukov. Sov. Phys. Semicond., 12, 387 (1978).

[3] F.F. Sizov, V.V. Teterkin, L.V. Prokofeva, E.A. Gurieva. Sov. Phys. Semicond., 14, 1063 (1980).

[4] A.A. Vinokurov, A.I. Artamkin, S.G. Dorofeev, T.A. Kuznetsova, V.P. Zlomanov. Inorg. Mater., 44, 576 (2008).

[5] E.P. Skipetrov, A.N. Golovanov, E.I. Slynko, V.E. Slynko. Low Temp. Phys., 39, 76 (2013).

[6] V.D. Vulchev, L.D. Borisova, K. Dimitrova. Phys. Status Solidi A, 97, K79 (1986).

[7] L.M. Kashirskaya, L.I. Ryabova, O.I. Tananaeva, N.A. Shirokova. Sov. Phys. Semicond., 24, 848 (1990).

[8] T. Story. Acta Phys. Pol. A, 94, 189 (1998).

[9] I.I. Ivanchik, D.R. Khokhlov, A.V. Morozov, A.A. Terekhov, E.I. Slyn'ko, V.E. Slyn'ko, A. de Visser, W.D. Dobrowolski. Phys. Rev. B, 61, R 14889 (2000).

[10] K.A. Kikoin, V.N. Fleurov. Transition Metal Impurities in Semiconductors: Electronic Structure and Physical Properties (Singapore, World Scientific, 1994).

[11] E.P. Skipetrov, O.V. Kruleveckaya, L.A. Skipetrova, E.I. Slynko, V.E. Slynko. Appl. Phys. Lett., 105, 022101 (2014).

[12] E.P. Skipetrov, O.V. Kruleveckaya, L.A. Skipetrova, A.V. Knotko, E.I. Slynko, V.E. Slynko. J. Appl. Phys., 118, 195701 (2015).

[13] E.P. Skipetrov, B.B. Kovalev, L.A. Skipetrova, A.V. Knotko, V.E. Slynko. J. Alloys Compd., 775, 769 (2019).

[14] E.P. Skipetrov, B.B. Kovalev, L.A. Skipetrova, A.V. Knotko, V.E. Slynko. Low Temp. Phys., 45, 201 (2019).

[15] G. Tan, F. Shi, S. Hao, H. Chi, L.-D. Zhao, C. Uher, C. Wolverton, V.P. Dravid, M.G. Kanatzidis. J. Am. Chem. Soc., 137, 5100 (2015).

[16] L. Wang, X. Tan, G. Liu, J. Xu, H. Shao, B. Yu, H. Jiang, S. Yue, J. Jiang. ACS Energy Lett., 2, 1203 (2017). 
[17] D.K. Bhat, U.S. Shenoy. Mater. Today Phys., 4, 12 (2018).

[18] G.J. Snyder, E.S. Toberer. Nature Materials, 7, 105 (2008).

[19] A.D. LaLonde, Y. Pei, H. Wang, G.J. Snyder. Mater. Today, 14, 526 (2011).

[20] X. Zhang, L.-D. Zhao. J. Materiomics, 1, 92 (2015).

[21] V.E. Slynko, W. Dobrowolski. Bull. Nat. University „Lviv Polytechnic“, Electronics, N 681, 144 (2010).

[22] E.I. Slynko, V.M. Vodopyanov, A.P. Bakhtinov, V.I. Ivanov, V.E. Slynko, W. Dobrowolski, V. Domukhowski. Visn. Lviv Polytec. Natl. Univ., Electronics, N 734, 67 (2012).

[23] V.I. Kaidanov, Yu.I. Ravich. Phys. Usp., 28, 31 (1985).

[24] E.P. Skipetrov, E.A. Zvereva, N.N. Dmitriev, A.V. Golubev, V.E. Slynko. Semiconductors, 40, 893 (2006).

[25] E.P. Skipetrov, N.A. Pichugin, E.I. Slyn'ko, V.E. Slyn'ko. Semiconductors, 47, 729 (2013).

[26] E. Skipetrov, E. Zvereva, L. Skipetrova, B. Kovalev, O. Volkova, A. Golubev, E. Slyn'ko. Phys. Status Solidi B, 241, 1100 (2004).

[27] M. Ratuszek, M.J. Ratuszek. J. Phys. Chem. Solidi, 46, 837 (1985).

[28] V.D. Vulchev, L.D. Borisova. Phys. Status Solidi A, 99, K53 (1987).

[29] E.P. Skipetrov, N.A. Pichugin, E.I. Slyn'ko, V.E. Slyn'ko. Low Temp. Phys., 37, 210 (2011).

[30] E.P. Skipetrov, O.V. Kruleveckaya, L.A. Skipetrova, V.E. Slynko. J. Appl. Phys., 121, 045702 (2017).

[31] G. Nimtz, B. Schlicht. In: Narrow-Gap Semiconductors [Springer Tracts in Modern Physics (Berlin-HeidelbergN.Y.-Tokyo, Springer Verlag, 1983) v. 98].

[32] R.N. Tauber, A.A. Machonis, I.B. Cadoff. J. Appl. Phys., 37, 4855 (1966).

[33] C.M. Jaworski, M.D. Nielsen, H. Wang, S.N. Girard, W. Cai, W.D. Porter, M.G. Kanatzidis, J.P. Heremans. Phys. Rev. B, 87, 045203 (2013).

[34] Z.M. Gibbs, H. Kim, H. Wang, R.L. White, F. Drymiotis, M. Kaviany, G.J. Snyder. Appl. Phys. Lett., 103, 262109 (2013).

[35] L.-D. Zhao, V.P. Dravid, M.G. Kanatzidis. Energy Environ. Sci., 7, 251 (2014).

Редактор Л.В. Шаронова

\section{Temperature coefficient of the movement of resonant iron level in $\mathbf{P b}_{1-x-y} \mathbf{S n}_{x} \mathbf{F e}_{y}$ Te alloys}

\author{
E.P. Skipetrov ${ }^{1,2}$, B.B. Kovalev ${ }^{1}$, L.A. Skipetrova ${ }^{1}$, \\ A.V. Knotko ${ }^{2}$, V.E. Slynko ${ }^{3}$ \\ ${ }^{1}$ Lomonosov Moscow State University \\ (Faculty of Physics), \\ 119991 Moscow, Russia \\ 2 Lomonosov Moscow State University \\ (Faculty of Materials Science), \\ 119991 Moscow, Russia \\ ${ }^{3}$ Institute of Materials Science Problems, National \\ Academy of Sciences of Ukraine, \\ 58001 Chernivtsy, Ukraine
}

Abstract The phase composition, the elemental composition and temperature dependences of the resistivity and of the Hall coefficient (temperature interval $4.2 \leq T \leq 300 \mathrm{~K}$, magnetic fields $B \leq 0.07 \mathrm{~T})$ in $\mathrm{Pb}_{1-x-y} \mathrm{Sn}_{x} \mathrm{Fe}_{y} \mathrm{Te}$ alloys are studied at variation of the tin and iron concentrations along the single-crystal ingots synthesized by the Bridgman-Stockbarger technique. The distributions of tin and iron along the ingots are obtained and anomalous temperature dependences of the Hall coefficient, associated with the pinning of the Fermi level by the resonant level of iron, located in the valence band, are found. The analysis of the experimental results is carried out in the framework of the model of the electronic structure rearrangement, involving the movement of the iron level relative to the top of the valence band with increasing tin concentration and temperature. The temperature coefficient of the movement of the level of iron with respect to the middle of the gap is determined and the possible diagrams for the rearrangement of the electronic structure with increasing temperature in the alloys with normal spectrum $(0.06 \leq x \leq 0.35)$ are proposed. 